\title{
Research on automatic loading \& unloading technology for vertical hot ring rolling process
}

\author{
Wang Xiaokai1 ${ }^{1,3, a}$, Wang Dehui ${ }^{1,2}$, Mei Xuesong ${ }^{3}$, Hua Lin $^{1,2}$, Dai Yutong ${ }^{4}$, and Wu Jian ${ }^{4}$ \\ ${ }^{1}$ Hubei Key Laboratory of Advanced Technology for Automotive Components, Wuhan University of \\ Technology, Wuhan 430070, China \\ ${ }^{2}$ Hubei Collaborative Innovation Center for Automotive Components Technology, Wuhan 430070, \\ China \\ ${ }^{3}$ College of Mechanical Engineering, Xi' an Jiaotong University, Xi'an 710049, China \\ ${ }^{4}$ Zhangjiagang Hailu Annular Forgings Co., Ltd., Zhang Jiagang 215626, China
}

\begin{abstract}
The automatic loading \& unloading technology is the key to the automatic ring production line. In this paper, the automatic vertical hot ring rolling (VHRR) process is taken as the target, the method of the loading \& unloading for VHRR is proposed, and the mechanical structure of loading \& unloading system is designed, The virtual prototype model of VHRR mill and loading \& unloading mechanism is established, and the coordinated control method of VHRR mill and loading \& unloading auxiliaries is studied, the movement trace and dynamic characteristic of the critical components are obtained. Finally, a series of hot ring rolling tests are conducted on the VHRR mill, and the production rhythm and the formed rings' geometric precision are analysed. The tests results show that the loading \& unloading technology can meet the high quality and high efficiency ring production requirement. The research conclusions have practical significance for the largescale automatic ring production.
\end{abstract}

\section{Introduction}

The medium-size rings are the fundamental parts which have been widely used in industry fields such as automobile, railway and machine tool [1,2]. Vertical hot ring rolling (VHRR) process is the advanced production technology for such rings. VHRR mill is the key equipment of VHRR process. At present, the VHRR mill has mainly realized automatic production, and numerical control (NC) VHRR mill has been applied in industry [3]. However, because of the high temperature of the ring blanks $\left(800 \sim 1150^{\circ}\right)$, the poor production environment and the short takt time $(3 \sim 6 \mathrm{~s} /$ piece $)[4,5]$, the loading $\&$ unloading of VHRR is still depends on manual operation during the continuous VHRR production, which increases the labor intensity and the production cost, influences the production efficiency and the quality stability of the ring products. This paper takes the NC VHRR mill and the VHRR production as the application objects. On the basis of the analysis of the VHRR process, the mechanical design scheme of the

\footnotetext{
${ }^{a}$ Corresponding author: wxk0919@163.com
}

This is an Open Access article distributed under the terms of the Creative Commons Attribution License 4.0, which permits unrestricted use, distribution, and reproduction in any medium, provided the original work is properly cited. 


\section{MATEC Web of Conferences}

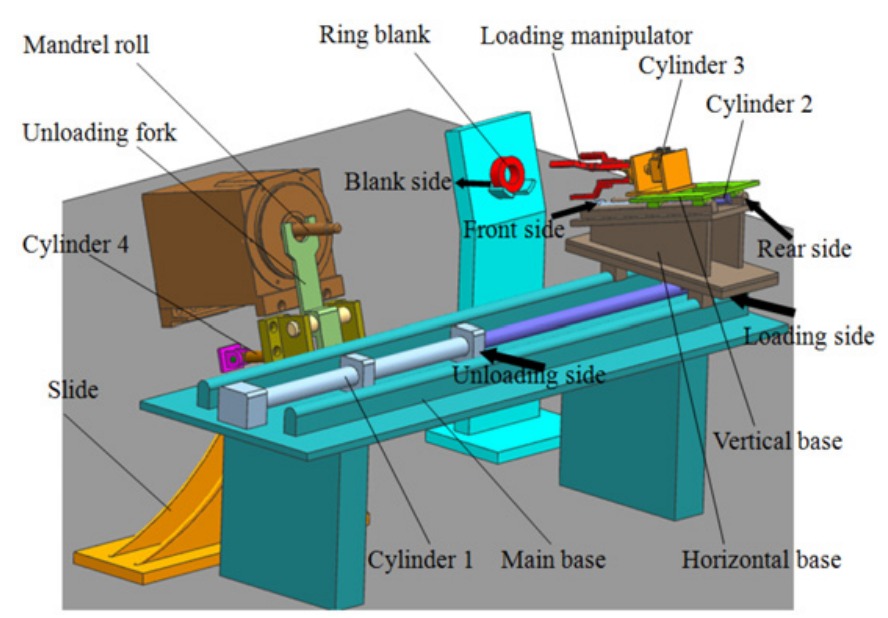

Figure 1. The structure diagram of the loading \& unloading auxiliaries.

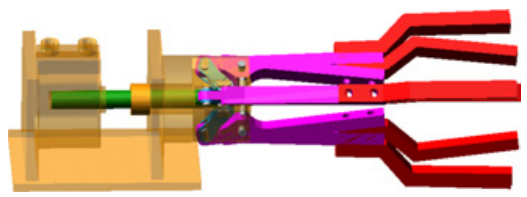

(a) The working principle of loading manipulator

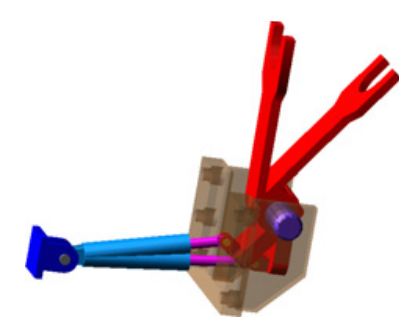

(b) The working principle of unloading fork

Figure 2. The working principle of the key components.

loading \& unloading auxiliaries is proposed. The working principle and procedure of the loading \& unloading auxiliaries are analyzed; the prototype of the loading \& unloading auxiliaries is manufactured, assembled and tested in the VHRR production line. The coordinated control of the loading \& unloading auxiliaries and VHRR mill is realized, and finally the automatic batch production of the ring products is achieved. The research result has practical significance to realize the VHRR automatic production, promote the quality stability and reduce the manufacturing cost of the ring products.

\section{The structure and principle of loading \& unloading auxiliaries}

To achieve the functions of holding the ring blanks reliably, passing the blanks through the mandrel roll accurately and unloading the rings smoothly. The designed auxiliaries contain several components: main base, horizontal base, vertical base, loading manipulator, unloading fork, slide and cylinders, as shown in Fig. 1.

Figure 2(a) shows the working principle of the loading manipulator. Cylinder 3 is utilized to drive the linkage to make the loading manipulator open and close, thus the blank feeding is realized. Figure 2(b) illustrates the working principle of unloading fork. Cylinder 4 is employed to drive the fork, and the fork can swing around the axle to-and-fro to push the formed ring from the mandrel roll to the slide. 


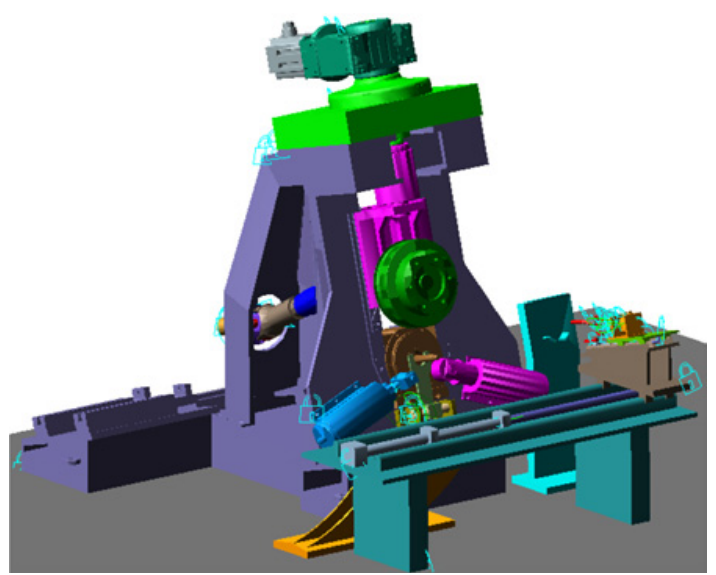

Figure 3. The virtual prototype with motion constraints.

The workflow of the loading \& unloading auxiliaries is as follows:

1) Before work, the horizontal base is at the loading side and the vertical base is at the rear side, as shown in Fig. 1. The loading manipulator is open.

2) When the ring blank is detected at the blank side as shown in Fig. 1, cylinder 2 will drive the vertical base to its front side.

3) The cylinder 3 will drive the loading manipulator close, and the ring blank will be clamped.

4) After clamping the ring blank, the vertical base will move to the rear side.

5) Cylinder 1 will pull the horizontal base to the unloading side, as shown in Fig. 1.

6) The control system detects that the horizontal base is at the unloading side, and the main roll of the VHRR mill is located at the origin. Cylinder 2 will drive the vertical base to the front side, and then the ring blank will pass through the mandrel roll.

7) When the vertical base arriving at the front side, cylinder 3 will push the loading manipulator open, and the ring blank will be placed on the mandrel roll, afterwards, the vertical base will return to the rear side.

8) When the vertical base is at the rear side, the main roll begins to move downwards, and the ring rolling process will be conducted.

9) After the ring rolling process finished, the main roll will return to the origin, then the cylinder 4 will drive the fork to swing to-and-fro, and the formed ring will unload from the mandrel roll to the slide.

10) At the same time with step 8) and 9), the horizontal base will move to the loading side to prepare for the next blank loading, the whole procedure completes and step 2) will be repeated.

\section{The motion simulation of the VHRR mill and loading \& unloading mechanism}

In order to validate the feasibility of the designed automatic loading \& unloading auxiliaries, the virtual prototype of VHRR mill and loading \& unloading auxiliaries is established in software ADAMS. The motion constraints are added to relative components. For example, the body of the VHRR mill and the main base should be fixed to the ground. The translational joint is applied on the horizontal base and vertical base. The revolute joint is used to the linkages of loading manipulator and unloading fork. The translation speed is added to each cylinder, as shown in Fig. 3. 


\section{MATEC Web of Conferences}

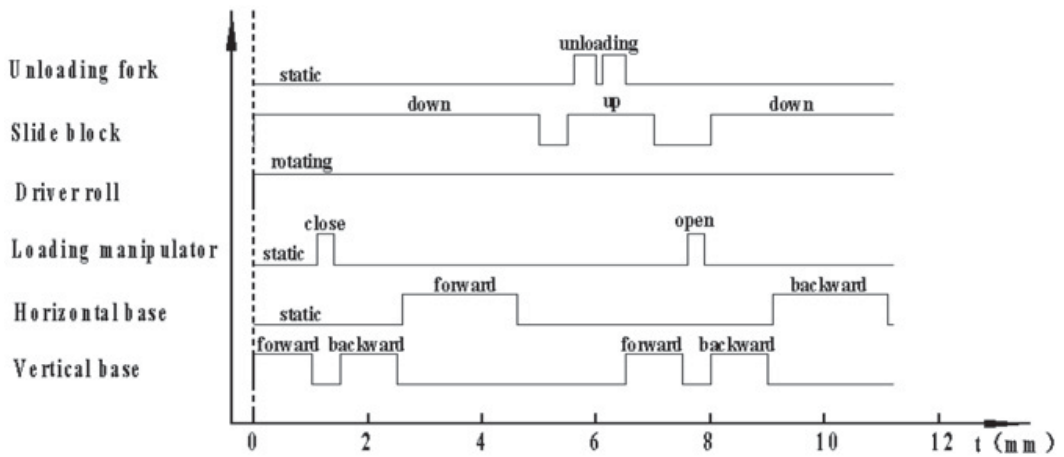

Figure 4. The sequence diagram of the main components.

Table 1. The movement parameters of components.

\begin{tabular}{|c|c|c|c|}
\hline Components & time $(S)$ & Velocity & displacement \\
\hline Vertical Base & $\begin{array}{l}0 \sim 1,1.5 \sim 2.5 \\
6.5 \sim 7.5,8 \sim 9\end{array}$ & $222 \mathrm{~mm} / \mathrm{s}$ & $222 \mathrm{~mm}$ \\
\hline Horizontal Base & $2.6 \sim 4.6,9.1 \sim 11.1$ & $357 \mathrm{~mm} / \mathrm{s}$ & $714 \mathrm{~mm}$ \\
\hline Cylinder 3 & $1.1 \sim 1.4,7.6 \sim 7.9$ & $10 \mathrm{~mm} / \mathrm{s}$ & $3 \mathrm{~mm}$ \\
\hline Driver Roll & $0 \sim 11.1$ & $4.71 \mathrm{rad} / \mathrm{s}$ & \\
\hline Driver Roll & $0 \sim 5$ & $\overline{-}$ & $100.5 \mathrm{~mm}$ \\
\hline Sliding block & $5.5 \sim 7$ & $67 \mathrm{~mm} / \mathrm{s}$ & $100.5 \mathrm{~mm}$ \\
\hline Cylinder 4 & $5.6 \sim 6,6.1 \sim 6.5$ & $90 \mathrm{~mm} / \mathrm{s}$ & $36 \mathrm{~mm}$ \\
\hline
\end{tabular}

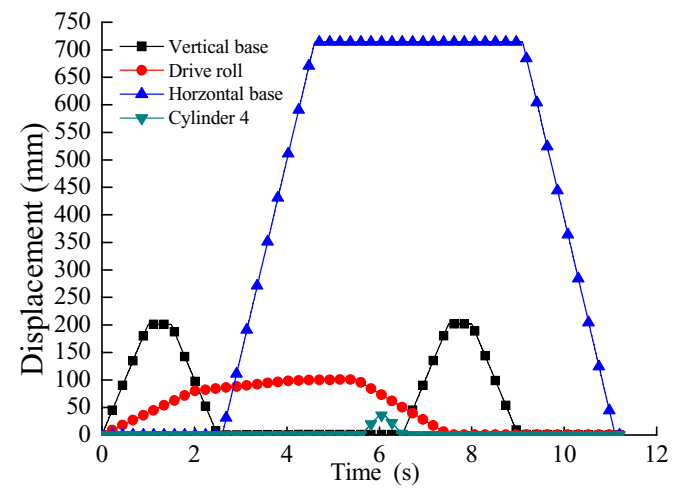

(a) Displacement-time curves of main components

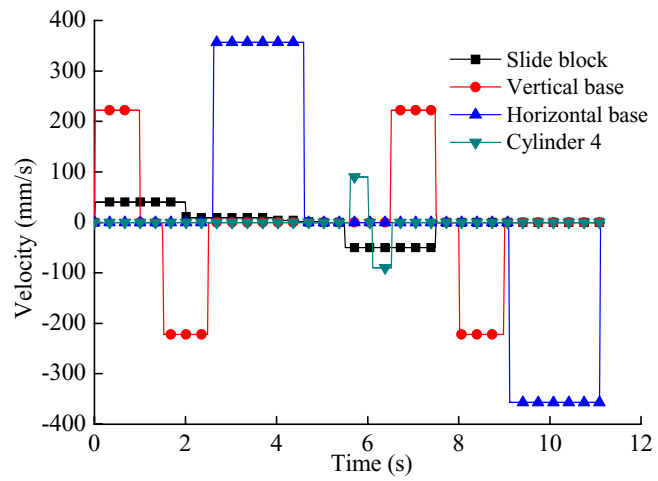

(b) Velocity-time curves of main components

Figure 5. Motion curves of the main component.

The sequence diagram of the main components in the virtual prototype model is shown in Fig. 4, and the parameters of each component are illustrated in Table 1.

Figure 5 shows the simulation results of the virtual prototype, the displacement and velocity curves of the vertical base, drive roll, horizontal base and cylinder 4 are obtained in ADAMS. It is found that there is no interference between the VHRR mill and the loading \& unloading auxiliaries both in time and space. 


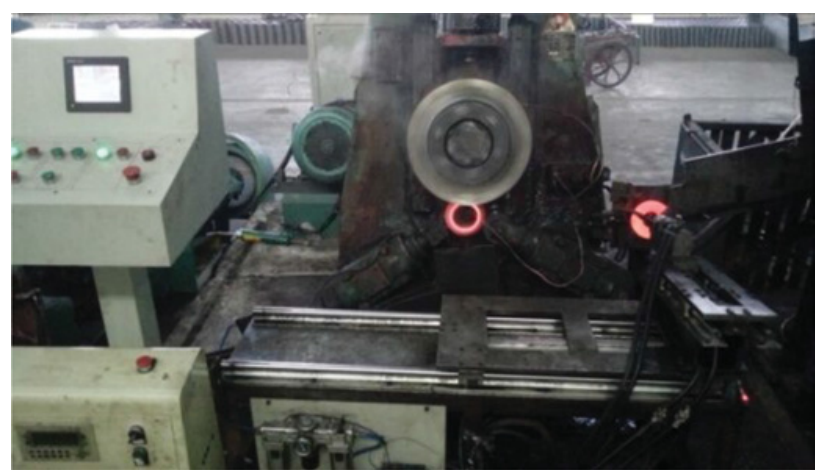

Figure 6. The automatic production line of VHRR.

Table 2. The results of tests.

\begin{tabular}{|c|c|c|c|}
\hline sample & Weight $(\mathbf{g})$ & outer diameter $(\mathbf{m m})$ & roundness error $(\mathbf{m m})$ \\
\hline 1 & 601.40 & 102.00 & 0.15 \\
\hline 2 & 603.80 & 102.11 & 0.24 \\
\hline 3 & 600.80 & 102.30 & 0.42 \\
\hline 4 & 602.00 & 102.05 & 0.27 \\
\hline 5 & 597.80 & 102.20 & 0.23 \\
\hline 6 & 597.20 & 102.30 & 0.55 \\
\hline 7 & 604.80 & 102.35 & 0.40 \\
\hline 8 & 603.20 & 102.35 & 0.42 \\
\hline 9 & 604.40 & 102.45 & 0.40 \\
\hline 10 & 601.00 & 102.38 & 0.20 \\
\hline
\end{tabular}

\section{The tests of the automatic loading \& unloading auxiliaries}

The loading \& unloading auxiliaries were manufactured and assembled. An automatic ring production line was formed of the loading \& unloading auxiliaries and HRM160 VHRR mill which is developed by Wuhan University of Technology. A series of hot ring rolling tests were carried out, and the whole production process is shown as Fig. 6.

The requirement of the formed rings is $101.92_{0}^{+1.0} \mathrm{~mm}$ in the tests. 10 formed rings were selected randomly, and their weight and dimensions were measured as shown in Table 2. It can be seen that the dimensions of the selected samples meet the design requirements. The maximum roundness error is $0.55 \mathrm{~mm}$ and the minimum value is $0.15 \mathrm{~mm}$. In addition, the automatic loading \& unloading auxiliaries designed have good adaptability to weight deviation of the ring blanks.

\section{Conclusions}

This research takes the NC VHRR mill as the application object, the design method and working principle of the automatic loading \& unloading auxiliaries are proposed. The kinematic analysis of the automatic loading \& unloading auxiliaries is conducted. The simulation results show that it has no interference between the VHRR mill and the loading \& unloading auxiliaries both in time and space. The VHRR mill and the loading \& unloading auxiliaries work harmoniously, and there is no impact on the production efficiency. Finally, the loading \& unloading prototype is manufactured. An automatic ring production line is formed of the prototype and the VHRR mill. Small batch production tests are 


\section{MATEC Web of Conferences}

conducted. The tests results show that the loading \& unloading prototype has good adaptability and can satisfy the requirements of automatic ring rolling production.

The authors would like to thank the National Natural Science Foundation for Young Scholars (No. 51205299), the Science and Technology Support Program of Hubei province (No. 2014BAA008), the Fundamental Research Funds for the Central Universities (No. 2014-IV-144) for the support given to this research.

\section{References}

[1] L. Hua, X.G. Huang, C.D. Zhu, Theory and technology of ring rolling (China Mechanical Industry Press, Beijing, 2001)

[2] G. Hirt, R. Kopp. Implementing a high accuracy multi-mesh method for incremental bulk metal forming. CIRP. Ann. Manuf. Tech. 56, 313 (2007)

[3] X.K. Wang, L. Hua, Modeling of on-line measurement for rolling the rings with blank size errors in vertical hot ring rolling process. Int. J. Adv. Manuf. Tech. 68, 257 (2013)

[4] J.M. Allwood, M. Julian, Tekkaya, The development of ring rolling technology. Steel. Res. Int. 76, $491(2005)$

[5] X.H. Han, L. Hua, X.K. Wang, Ring blank design and its effect on combined radial and axial ring rolling. Int. J. Adv. Manuf. Tech. 72, 1161 (2014) 